\title{
DEUTERIUM IN THE DIFFUSE INTERSTELLAR MEDIUM
}

\author{
R. FERLET \\ Institut d'Astrophysique de Paris, CNRS \\ 98 bis Boulevard Arago, 75014 Paris, France
}

\begin{abstract}
We review the observational status in evaluating the interstellar deuterium abundance and show that the most reasonable value is of the order of $10^{-5}$. Although in general agreement with the standard Big Bang nucleosynthesis, the situation is still unclear and deserves much more observations.
\end{abstract}

\section{Introduction}

It is largely accepted that the main site of formation of the light elements ${ }^{2} \mathrm{H},{ }^{3} \mathrm{He},{ }^{1} \mathrm{He}$, ${ }^{7} \mathrm{Li}$ is during the first few minutes of the Universe, in the frame of the so-called standard Big Bang nucleosynthesis. Among these, deuterium is the most sensitive to primordial conditions, and determining its primordial abundance is therefore of great cosmological significance (see e.g. [1]).

Since the formation of the Galaxy, the evolution of the deuterium abundance $X_{1}$ ) is relatively straightforward because it is simply burned when passing through stars. It is predicted that this Galactic astration should decrease $X_{I}$ by no more than a factor of $\sim 2$, along with a negative gradient from the Galactic center to the edge small enough to expect a constant $\mathrm{D} / \mathrm{H}$ ratio in the solar neighbourhood [2]. Although this simplest approach has not yet been proved to be wrong, one should keep in mind that several poorly known parameters and/or unknown pregalactic events could strongly affect these predictions.

\section{Deuterium Observations}

Prior to 1972, deuterium was only measured on the Earth, either in the ocean or inside meteorites, at $\mathrm{a} \sim 1.510^{-4}$ level. Then, it was observed indirectly through ${ }^{3} \mathrm{He}$ in the solar wind or directly in planets, in the interstellar medium through deuterated molecules (see respectively Owen and Tielens in these proceedings) or through its atomic form, and in the atmosphere of massive stars.

Concerning stars, it has been shown by [3] that in Canopus, $\mathrm{D} / \mathrm{H} \lesssim 5.510^{-7}$, while in the main sequence star $\alpha \mathrm{Pav}, \mathrm{D} / \mathrm{H} \lesssim 10^{-5}[4]$. This latter result implies that deuterium was either also destroyed in a main sequence star, in contradiction with existing model predictions, or that the present value of $\mathrm{D} / \mathrm{H}$ is at most $10^{-5}$ with the important consequences which will be discussed later. 
Although extremely difficult, attempts to observe directly interstellar deuterium atoms through their hyperfine transition at $91.6 \mathrm{~cm}$ are still underway. Following the original approach of [5], [6] and [7] did not confirm the deuterium line in absorption in the direction of the Galactic center used as a background source and set $\mathrm{D} / \mathrm{H}<510^{-4}$. More recently, [8] searched for deuterium emission in the anti-center direction and found a marginal detection yielding a present $\mathrm{D} / \mathrm{H}<610^{-5}$.

The more precise evaluations of the deuterium abundance are made in the diffuse interstellar medium through the observation of the Lyman lines of both $\mathrm{H}$ and $\mathrm{D}$ seen in absorption in the spectra of early $B$ and $O$ type fast rotating stars used as background sources. This was one of the major accomplishments of the Copernicus satellite which operated in the far UV at high spectral resolution $\left(15 \mathrm{~km} \mathrm{~s}^{-1}\right)$ from 1972 to 1980 (see fig.1a, ref. [9] to [16]).

From about a dozen of lines of sight observed, a scatter of more than a factor of 4 clearly shows up, which it is extremely difficult to attribute to instrumental and/or analysing errors only in view of the quality of the Copernicus data and the number of independent spectral lines used in the Lyman series (up to 5). However, these variations may not be due to deuterium, since $\mathrm{H}$ atoms blue shifted by about $80 \mathrm{~km} \mathrm{~s}^{-1}$ will produce the same spectral signature.

At least two sources of high velocity $\mathrm{HI}$ can provide such a contamination : shocks in the interstellar medium and structures in the stellar winds themselves of the hot target stars. By eliminating the lines of sight which seems to be polluted in that way [17], as well as those with lower quality data or for which only one Lyman line was observed, we finally end up with an average $\mathrm{D} / \mathrm{H}$ value toward $\epsilon$ Ori, $\delta$ Ori, $\gamma$ Cas and $\lambda$ Sco (see fig. 1a) of $8.610^{-6}$. Even more carefully, $\gamma$ Cas is well known to exhibit a shell-like activity with a $\mathrm{X}$-ray emission and should be also rejected. Thus, the present day $\mathrm{D} / \mathrm{H}$ ratio in the interstellar medium is $710^{-6}$ in number or $\mathrm{X}_{\mathrm{I}} \sim(1 \pm 0.5) 10^{-5}$ by mass.

The interstellar deuterium can also be observed toward cool nearby stars, in absorption against the Lyman $\alpha$ stellar emission (see fig.1b, ref. [18] to [26], which includes results both from the Copernicus and the IUE satellites; IUE is still in operation since 1978 but with a lower spectral resolution of $30 \mathrm{~km} \mathrm{~s}^{-1}$ ). In spite of the necessary modelling of the stellar emission for defining the continuum for the interstellar absorption and of the eventually multiple velocity components on the line of sight not resolved with IUE, the more recent and detailed study by [26] toward $\alpha$ Aur and $\lambda$ And (fig.1b) confirms the high $\mathrm{D} / \mathrm{H}$ ratios found in the local interstellar medium, indicating that this ratio might change by possibly a factor of four over scales as small as few parsecs. If these variations are confirmed to be a local phenomenon, they should not affect the "average" $\mathrm{D} / \mathrm{H}$ value derived above over larger scales.

In conclusion, following the standard Galactic evolution model, the pre-Galactic deuterium abundance is :

$$
X_{\mathrm{Dp}} \sim 2 \pm 110^{-5} \quad \text { by mass. }
$$

\section{Discussion}

The comparison of this result, along with other light elements abundance evaluations, with primordial nucleosynthesis predictions leads to a reasonably good agreement showing that the baryons contribute between 0.02 and 0.11 to the critical energy density of the Universe, in the frame of the standard Big Bang model calculations [27]. 


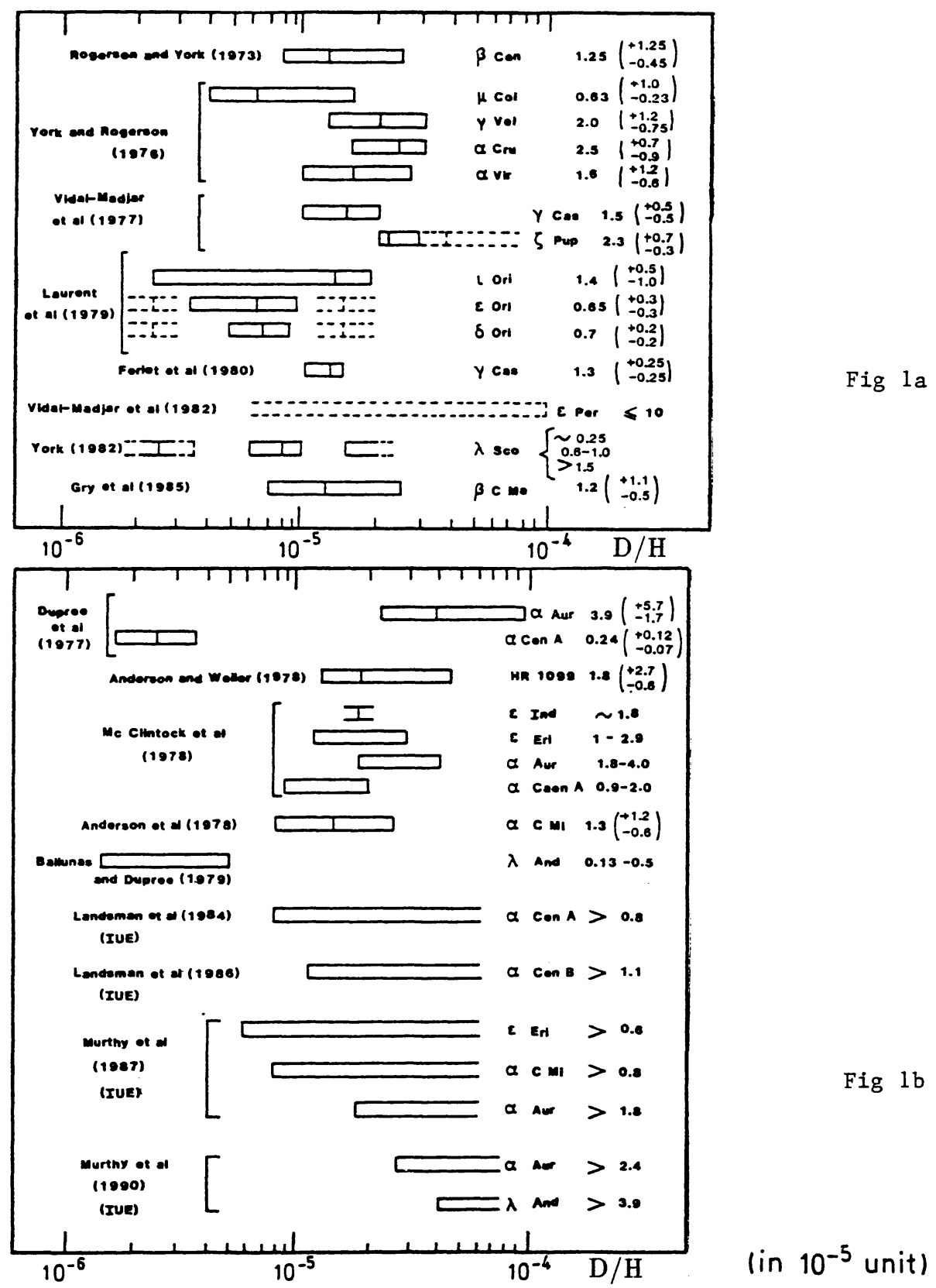

Figure 1. Observations of the $\mathrm{D} / \mathrm{H}$ ratio in the diffuse interstellar medium through Lyman lines in absorption toward hot stars in the range 100-1000 pc (Fig.1a, ref. [9] to [16]), or toward very nearby cool stars (Fig.1b, ref. [18] to [26]). The average interstellar $\mathrm{D} / \mathrm{H}$ ratio is around $10^{-5}$, while local variations seem to be present (see text). 
It has to be stressed that this model has now reach a real maturity, being able to produce correct predictions in the field of particle physics. In effect, it was easily shown by [28] that probably only three families of neutrinos were compatible with the observed helium abundance, a fourth one being barely acceptable. LEP experiments at CERN beautifully confirmed that prediction [29].

This should now lead to a more detailed comparison between the predicted and the observed abundances in order to detect possible fine tuning disagreement which might be the signature of still unclear issues. Among them, the Galactic evolution is certainly poorly known, as it is shown by [30] through the history of the light element abundance measurements : in spite of their large variations with instrumental progress, it was invariably concluded to a good agreement with the predictions.

The $\mathrm{D}$ constraint can now be replaced by the ${ }^{3} \mathrm{He}+\mathrm{D}$ one since the sum is measured in the solar wind [31]. This is the unique site of measurement, and it seems to fit the model. We have shown here that more numerous deuterium observations have brought difficulties. Much more are needed to rise interesting constraints, as is already the case for ${ }^{4} \mathrm{He}$ and ${ }^{7} \mathrm{Li}$, either at $92 \mathrm{~cm}$ or in the UV and visible wavelength ranges. Thus, Lyman lines will be seen at different redshifts with the Hubble Space Telescope and the FUSE-LYMAN explorer, and will perhaps be detected in "primordial" clouds in absorption toward quasars [32] accessible to large ground-based telescopes. We may at the end reach the solution.

\section{References}

[1] Vidal-Madjar, A., 1982, in "Diffuse Matter in Galaxies", Audouze et al. (eds), NATO ASI Series, $\mathrm{N}^{\circ} 110,57$

[2] Audouze, J. and Tinsley, B.M., 1976, Ann. Rev. Astron. Ap., 14, 43

[3] Ferlet, R., Dennefeld, M. and Spite, M., 1983, Astron. Astrophys., 124, 172

[4] Vidal-Madjar, A., Ferlet, R., Spite, M. and Coupry, M.F., 1988, Astron. Astrophys., 201, 273

[5] Césarsky, D.A., Moffet, A.T. and Pasachoff, J.M., 1973, Astrophys. J. Letters, 180, L1

[6] Sarma, N.V.G. and Mohanty, D.K., 1978, M.N.R.A.S., 184, 181

[7] Anantharamaiah, K.R. and Radhakrishnan, V., 1979, Astron. Astrophys. Letters, 79, L9

[8] Blitz, L. and Heiles, C., 1987, Astrophys. J. Letters, 313, L95

[9] Rogerson, J.B. and York, D.G., 1973, Astrophys. J. Letters, 186, L95

[10] York, D.G. and Rogerson, J.B., 1976, Astrophys. J., 203, 378

[11] Vidal-Madjar, A., Laurent, C., Bonnet, R.M. and York, D.G., 1977, Astrophys. J., 211, 91

[12] Laurent, C., Vidal-Madjar, A. and York, D.G., 1979, Astrophys. J., 229, 923

[13] Ferlet, R., Vidal-Madjar, A., Laurent, C. and York, D.G., 1980, Astrophys. J., 242, 576

[14] Vidal-Madjar, A., Laurent, C., Gry, C., Bruston, P., Ferlet, R. and York, D.G., 1983, Astron. Astrophys., 120, 58

[15] York, D.G., 1983, Astrophys. J., 264, 172

[16] Gry, C., York, D.G. and Vidal-Madjar, A., 1985, Astrophys. J., 296, 593

[17] Gry, C., Lamers, H.J.G.L.M. and Vidal-Madjar, A., 1984, Astron. Astrophys., 137, 29

[18] Dupree, A.K., Baliunas, S.L. and Shipman, H.L., 1977, Astrophys. J., 218, 361

[19] Anderson, R.C. and Weiler, E.J., 1978, Astrophys. J., 224, 143 
[20] McClintock, W., Henry, R.C., Linsky, J.L. and Moos, H.W., 1978, Astrophys. J., 225, 465

[21] Anderson, R.C., Henry, R.C., Moos, H.W. and Linsky, J.L., 1978, Astrophys. J., 226, 883

[22] Baliunas, S.L. and Dupree, A.K., 1979, Astrophys. J., 227, 870

[23] Landsman, W.B., Henry, R.C., Moos, H.W. and Linsky, J.L., 1984, Astrophys. J., 285, 801

[24] Landsman, W.B., Murthy, J., Henry, R.C., Moos, H.W. Linsky, J.L. and Russel, J.L., 1986, Astrophys. J., 303, 791

[25] Murthy, J., Henry, R.C., Moos, H.W., Landsman, W., Linsky, J.L., Vidal-Madjar, A. and Gry, C., 1987, Astrophys. J., 315, 675

[26] Murthy, J., Henry, R.C., Moos, H.W., Vidal-Madjar, A., Linsky, J.L. and Gry, C., 1990, Astrophys. J., 356, 223

[27] Olive, K.A., Schramm, D.N., Steigman, G. and Walker, T.P., 1990, Phys. Letters, B236, 454

[28] Yang, J., Schramm, D.N., Steigman, G. and Rood, R.T., 1979, Astrophys. J., 227, 697

[29] L3 Collab., Adeva, B., et al., 1989, Phys. Letters, B231, 509

ALEPH Collab., Decamp, D., et al., 1989, Phys. Letters, B231, 519

OPAL Collab., Akrawy, M.Z., et al., 1989, Phys. Letters, B231, 530

DELPHI Collab., Aarnio, P., et al., 1989, Phys. Letters, B231, 539

[30] Vidal-Madjar, A., 1990, in COSPAR XXVIII, The Hague

[31] Bochsler, P., Geiss, J. and Maeder, A., 1990, Solar Physics, 128, 203

[32] Webb, J.K., Carswell, R.F., Irwin, M.J. and Penston, M.V., 1991, "On measuring the deuterium abundance in QSO absorption systems", preprint. 


\section{QUESTIONS AND ANSWERS}

S.P.Tarafdar: What are the equivalent width of Hydrogen Lyman- $\alpha$ forest lines? What will be the expected equivalent width of corresponding deuterium Ly- $\alpha$ forest lines and, can we observe them with our present day capability?

R.Ferlet: Using existing spectrographs at a resolution of $\sim 10 \mathrm{~km} / \mathrm{s}$ on a ground-based $4 m$ telescope, with an integration time of about 10 hours on a $m_{r} \sim 17.5$ quasar of redshift $>2.6$ in order to observe the first 5 lines of the Lyman series with a continuum $S / N$ ratio $\sim 15$, the lowest HI column density in a "Ly $\alpha$ forest" absorption line required for detecting DI at $\mathrm{Ly} \alpha$ if $D / H=10^{-4}$ is $\sim 10^{17}$ atoms $\mathrm{cm}^{-2}$ (assuming a $5 \sigma$ detection limit or a limiting equivalent width of about $70 \mathrm{~m} \dot{A}$ ). In that case, the DI Ly $\alpha$ equivalent width is predicted to be $\sim 110 \mathrm{m \dot {A }}$. The highest HI column densities seen in QSO absorption clouds are $\sim 10^{21} \mathrm{~cm}^{-2}$; in that case, deuterium (still if $D / H=10^{-4}$ ) will be detectable in Ly $\gamma, \delta$ and $\epsilon$ with equivalent widths $\sim 300 \mathrm{~m} \dot{A}$. Note that in all cases, the DI line is blended with the HI one. These simulations by Webb et al. (1991; preprint) are for $b=25 \mathrm{~km} / \mathrm{s}$. For $b=35 \mathrm{~km} / \mathrm{s}$, or if $D / H<10^{-4}$, the detection constraints are more stringent.

J.C.Pecker: You have insisted quite convincingly, in my opinion, upon the "pollution" which is endeavored by the $\mathrm{D} / \mathrm{H}$ ratio from place to place, and time to time, in the evolution of a galaxy. On the other side, Shapiro, before you, has stressed, as you did also, the bad knowledge of the early stages of the galactic evolution, which may imply highly intense UV sources so far and unknown evolutive processes. I would like therefore to insist upon the use (and abuse!) of the adjective "primordial" or "primaeval", when refering to varius determinations of abundances, be it $\mathrm{D} / \mathrm{H}$ or, say, $\mathrm{He}^{4} / \mathrm{H}$ or else. It seems to me that one should, each time, to be clearer: some determinations lead to "primordial" abundances at the time scale of the solar system; some others aim at the ( different) "primordial" abundances at the time scale of the lifetime of our Galaxy; whether some refer really to the scale time of the universe is very much in doubt, to my eyes. On line with this comment, I would add the following question: what do predict the models differing from the standard Big Bang, and I am thinking of $\Lambda \neq 1$, of inflationary models,etc. which constitute a very different "new Big Bang" from what is known as the standard one? What abundances can the "new Big(s) Bang(s)" predict?

R.Ferlet: Amongst non-standard Big Bang nucleosynthesis models, inhomogeneous ones inspired from the quark-hadron phase transitions have been the subject of recent and active research. However, they involve several free parameters and are still controversial. Nevertheless, they could become especially interesting if the observed "primordial" helium abundance is derived to be smaller than currently thought, if the "primordial" ${ }^{7} \mathrm{Li}$ abundance is found to be higher than the abundance measured in very metal-poor Population II stars (i.e. if it is demonstrated that surface lithium has been destroyed in these stars), if there is a significant cosmological yield to elements as ${ }^{\circ} \mathrm{Be}$, and/or if it exists a neutrino with a mass of $17 \mathrm{keV}$.

L.Blitz: Two comments on the 92-cm line work: (1) In the work I published with Heiles, done with the help of a number of people in the room, we were careful not to claim a detection, in spite of the suggestiveness of the spectrum. (2) More important, the DI line has now been detected by Heiles and McCollough toward Cas $A$ in a completely convincing spectrum that has now been confirmed. The problem is that it is in a difficult region to interpret since an atomic line has been observed in a clump of both atomic and molecular gas, where there is likely to have been some stellar processing, but a preliminary estimate gives $D / H \sim 1.5 \times 10^{-5}$. 